\title{
Application of Exit-Plane Wave Function Images in High-Resolution Transmission Electron Microscopy for Compositional Analysis of III-V Semiconductor Interfaces
}

\author{
K. Mahalingam, ${ }^{*}$ K. G. Eyink, ${ }^{*}$ G. J. Brown, ${ }^{*}$ D. L. Dorsey, ${ }^{*}$ C. F. Kisielowski, ${ }^{* *}$ and A. Thust ${ }^{* * *}$ \\ *Air Force Research Laboratory, Materials \& Manufacturing Directorate, Wright-Patterson AFB, \\ OH 45433-7707, USA.
}

N* National Center for Electron Microscopy,Lawrence Berkeley National Laboratory, Berkeley,
CA 94720 , USA.
${ }^{* * *}$ Institut für Festkörperforschung, Forschungszentrum Jülich GmbH, D-52425 Jülich, Germany

Existing methods based on high-resolution transmission electron microscopy (HRTEM) for compositional analysis of III-V semiconductor interfaces are applicable only to ternary systems [14]. Furthermore, they require optimal specimen-thickness and imaging conditions which depend on the material system under investigation. In this work we investigate the applicability of exit-plane wave function (EPWF) images, retrieved from HRTEM images, for quantitative chemical analysis of III-V semiconductor interfaces. By using the focus variation technique for EPWF retrieval [5] in combination with factorial analysis of correspondence for quantitative analysis [6], we show that compositional profiles along the group-III and group-V sublattices can be independently extracted. The present approach is more general, enabling compostional analysis of other III-V semiconductor heterostructures, such as InGaSb/InAs and InGaP/GaAs, which exhibit intermixing in both group-III and group-V sublattices. Application of the proposed method to the analysis of interfaces in epitaxially grown $\mathrm{Al}_{\mathrm{x}} \mathrm{Ga}(1-\mathrm{x}) \mathrm{As} / \mathrm{GaAs}$ and $\mathrm{In}_{\mathrm{x}} \mathrm{Ga}_{(1-\mathrm{x})} \mathrm{Sb} / \mathrm{InAs}$ heterostructures yielded compositional sensitivities with standard deviations equal to $0.06\left(\mathrm{x}_{\mathrm{Al}-\mathrm{Ga}}\right)$ and $0.08\left(\mathrm{x}_{\mathrm{In}-\mathrm{Ga}}\right.$ and $\left.\mathrm{x}_{\mathrm{As}-\mathrm{Sb}}\right)$, respectively. The validity of this approach is verified by an image simulation study performed on model interfaces with abrupt and linear grading in the interface composition profiles.

\section{References}

[1] A. Ourmazd et al., Ultramicroscopy 34 (1990) 237.

[2] S. Thoma and H. Cerva, Ultramicroscopy 38 (1991) 265.

[3] A. Rosenauer et al., Ultramicroscopy 72 (1998) 121.

[4] K. Tillmann et al., Ultramicroscopy 93 (2002)123.

[5] W. M. J. Coene et al., Ultramicroscopy 64 (1996) 167.

[6] P. Trebbia and N. Bonnet, Ultramicroscopy 34 (1990) 165.

[7] This work was supported by U. S. Air Force contract F33615-03-D-5801 under the sponsorship of the Materials Directorate, Air Force Research Laboratory, AFRL/MLPS, at Wright-Patterson Air Force Base, Ohio, USA. 

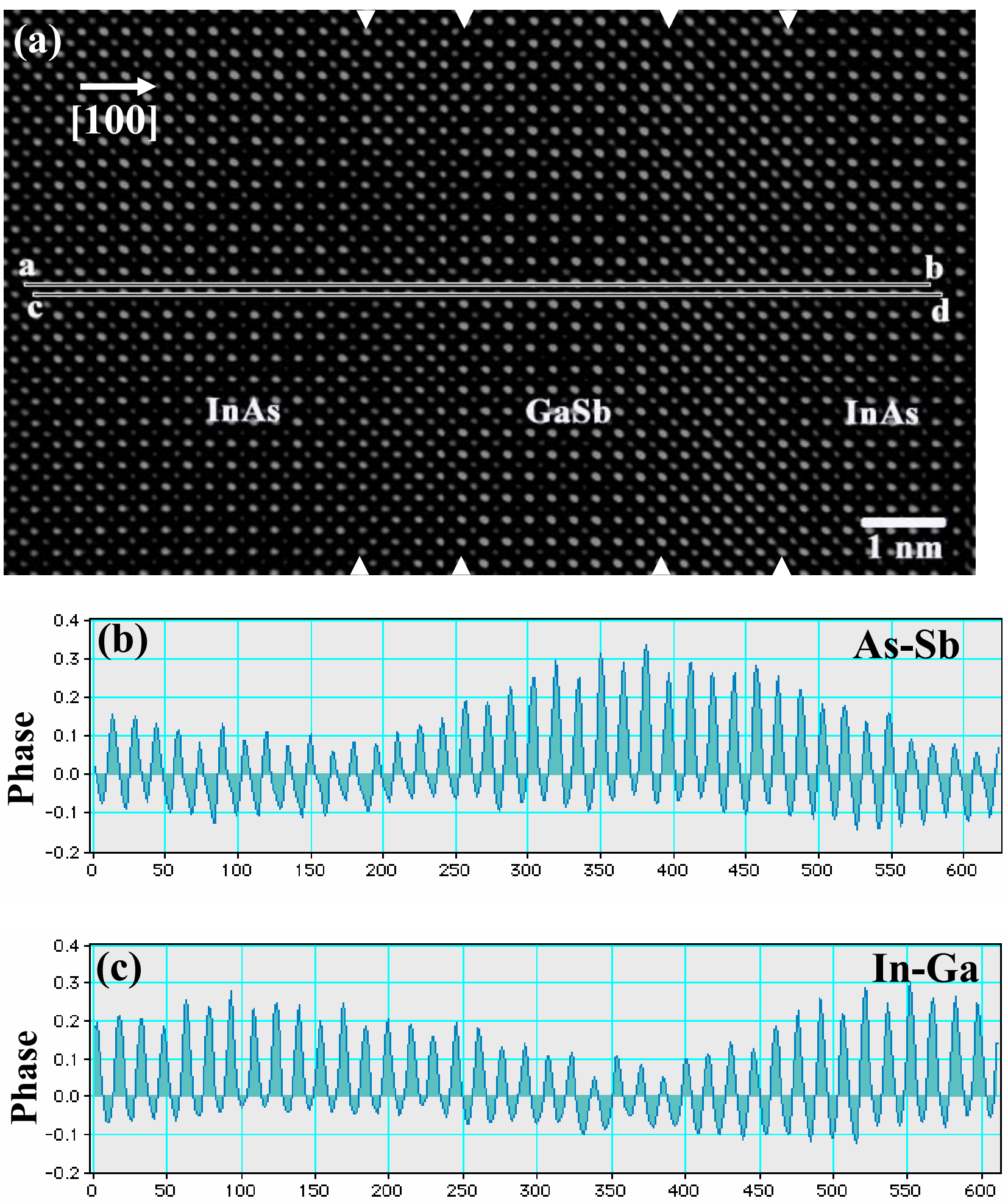

Fig. 1 (a) Exit-plane wave function phase component image of an InAs/GaSb/InAs structure, wherein the arrows mark interfacial regions adjacent to the two interfaces, (b) a line profile across the As-Sb sublattice, denoted by the line a-b in (a), and (c) a line profile across an adjacent In-Ga sublattice, denoted by the line c-d in (a). 